Localized electro-thermal processing: a new route to the patterning of magnetic recording media

This article has been downloaded from IOPscience. Please scroll down to see the full text article.

2010 Nanotechnology 21505303

(http://iopscience.iop.org/0957-4484/21/50/505303)

View the table of contents for this issue, or go to the journal homepage for more

Download details:

IP Address: 144.173.204.17

The article was downloaded on 15/08/2012 at 13:51

Please note that terms and conditions apply. 


\title{
Localized electro-thermal processing: a new route to the patterning of magnetic recording media
}

\author{
M M Aziz ${ }^{1}$, D M Newman, A Sidwell, M L Wears and C D Wright \\ College of Engineering, Mathematics and Physical Sciences, University of Exeter, \\ Harrison Building, Exeter EX4 4QF, UK \\ E-mail: M.M.Aziz@exeter.ac.uk
}

Received 6 August 2010, in final form 1 November 2010

Published 22 November 2010

Online at stacks.iop.org/Nano/21/505303

\begin{abstract}
Previous reports have detailed the fabrication of media able to support high density magnetic recording in both longitudinal and perpendicular formats by the global rapid thermal processing of sputtered non-magnetic precursor films. During processing in this manner a magnetic element is released from its nitride and agglomerates to form a random near mono-dispersion of magnetic nano-particles. Here we explore, primarily through modelling and simulation, the feasibility of processing similarly formulated precursor media not globally but locally. We investigate the potential of using conducting nano-probe tips to produce, via electro-thermal (Joule) heating, a nano-patterned recording medium in the form of regular arrays of magnetic islands in a non-magnetic host. In the first instance we concentrate on the simplest cobalt based precursor medium for which both initial simulation and experimental studies indicate the formation of magnetic islands with dimensions of the order of the tip diameter; this is relatively straightforward. The results signify that if practical production scenarios can be devised to produce technologically significant areas of recording media by the rapid multi-probe repetition of this technique, then processing in this manner offers a promising route to areal recording densities of perhaps 5 Terabit/in ${ }^{2}$ even with the simplest cobalt media. We also note that the electro-thermal processing method is potentially extendable to the production of a wide variety of magnetic materials (e.g. PtCo, FeCo, NiFe alloys) and, applied via electrical nano-imprinting type techniques, to the production of a wide variety of patterned structures.
\end{abstract}

\section{Introduction}

Following the recent adoption of perpendicular mode recording, the global recording industry is simultaneously studying several potential solutions to the problem of the super-paramagnetic limit and its apparent constraint on the attainment of areal densities of 1 Terabit/in ${ }^{2}$ and beyond. The first of these retains the current many-particles-perbit concept and allows the grain size to reduce whilst overcoming the attendant loss of thermal stability by the adoption of high anisotropy alloys such as PtCo or PtFe [1]. In comparison, the alternative solution requires neither increase in anisotropy nor reduction in particle volume but offers dramatic increases in areal density by moving to a

\footnotetext{
1 Author to whom any correspondence should be addressed.
}

one-bit-per-particle recording format [2]. Although apparently offering ways forward, each approach still presents formidable technical challenges to commercial realization. Whilst the adoption of high anisotropy alloys allows the use of very fine grain media, it is achieved at the expense of increasing the coercivity beyond the write field attainable in conventional heads even when assisted by soft magnetic under-layers. This approach therefore awaits the development and cost-effective commercialization of energy-assisted recording head and media technologies such as heat-assisted magnetic recording (HAMR) [3] and microwave-assisted magnetic recording (MAMR) [4]. In the case of the one-bit-per-particle approach, the major technological challenge (apart from bit addressing) resides in the difficulty of producing regularly patterned arrays of identically dimensioned magnetic nano-dots over technologically significant areas. 


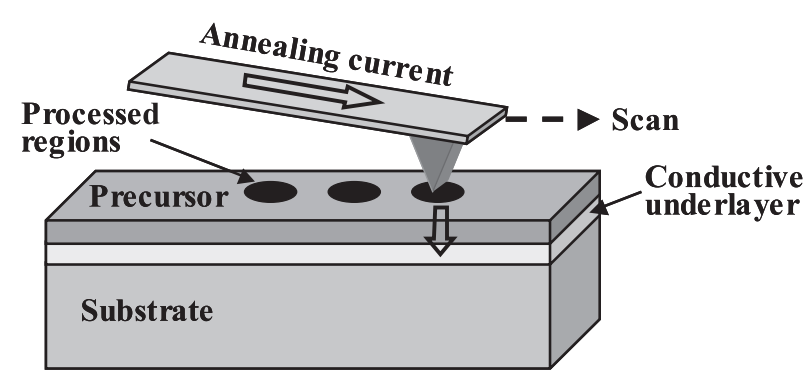

Figure 1. Schematic of the basic concept of localized nano-probe, electro-thermal processing of a precursor layer to produce isolated magnetic islands.

In previous reports [5-10] we described the development of a fabrication process for the production of homogenized, near mono-disperse, nano-particulate recording media by the global rapid thermal processing of sputtered non-magnetic precursor layers based on cobalt nitride. Both Co and PtCo media were produced by this process and demonstrated the ability to support ultra-high-density recording under the manyparticle-per-bit regime in the longitudinal and perpendicular modes respectively. In our last report [10] on this work we also commented that further increases in the storage potential of this media fabrication process should be achievable if it were possible to process the precursor material not globally but locally at the nano-scale to realize a regularly patterned medium able to support recording in the one-particle-per-bit format. Magnetic islands produced in this manner would have to be smaller than the single domain limit and isolated from each other by non-magnetic unprocessed precursor. It is considered that this might most easily be achieved by using electrical current pulses from a conducting atomic force microscope (AFM) tip to locally process the precursor film by Joule heating, as shown schematically in figure 1. Of course to then exploit this in any practical application such as a recording system will require replacement of the single tip shown with a large $2 \mathrm{D}$ array of identical tips. In this paper we construct a model of the localized electro-thermal transformation of the precursor medium that allows the potential of this form of processing to be explored by running simulations which are then compared with the simplest experimental studies.

\section{Modelling rational and methodology}

We confine this initial study to precursor films of the simple cobalt nitride system as there are numerous experimental studies of the preparation and processing of such films in the literature [11-15] that confirm our own understanding of the mechanisms by which cobalt nitride dissociates to cobalt via the release of nitrogen. All agree that the dissociation process is complex, involves a number of reaction steps and that the transition temperature, although centred around $573 \mathrm{~K}$, can occur anywhere between 500 and $670 \mathrm{~K}$ depending on the specific thermal processing conditions applied and in particular on the heating rates. This complexity renders it immediately obvious that a model based only on the assumption that the $\mathrm{Co}-\mathrm{N}$ system translates to the cobalt phase with the evolution of nitrogen when the localized temperature becomes equal to or greater than a specific transition temperature without consideration of the reaction kinetics, and in particular the reaction enthalpy which is known to be exothermic [10], is unlikely to be satisfactory for our purpose. Any attempt at developing a full and complete description of the process is, however, hampered by the lack of thermodynamic and kinetic information both about the process as a whole and each of the specific individual reaction steps involved. Our approach therefore recognizes these difficulties and aims to produce a simple but practical model that captures the fundamentals of the thermodynamics and kinetics involved in the dissociation reaction occurring during nano-scale local heating by the probe whilst remaining sufficiently sophisticated to allow reliable prediction of the shapes and volumes of the cobalt structures that form in the locally processed regions. It is also intended to allow subsequent study of the thermal stability of the formed cobalt structures during reheating.

We take as our starting point a simplified model of the dissociation reaction based on the balance equation:

$$
(1-x) \operatorname{CoN}(\mathrm{s}) \leftrightarrow x \operatorname{Co}(\mathrm{s})+\frac{x}{2} \mathrm{~N}_{2}(\mathrm{~g})
$$

in which $x$ is the molar fraction of CoN decomposed, the phase of a component is signified solid or gas by ( $\mathrm{s}$ ) or $(\mathrm{g})$ respectively and we justify this approach on the grounds that:

(i) thermodynamic parameters are obtainable for $\mathrm{CoN}$ by predictions from other functions (i.e. enthalpy of formation, entropy and Gibbs free energy),

(ii) the thermodynamics indicate the feasibility of following this route as will be shown later and

(iii) according to Hess's law [16], the enthalpy of the reaction in (1) includes all the energies of the intermediary states or reactions during the dissociation process.

The authors are unaware of any reported values for the reaction kinetics of the different phases to allow more detailed modelling of the multi-step reaction.

\subsection{Obtaining the thermodynamic parameter}

Thermodynamic parameters for $\mathrm{Co}-\mathrm{N}$ systems are scarce $[17,18]$ and no experimental data are available for CoN. We overcome this by exploiting the predictive approach of Guillermet and Jonsson [19] to the Co-N system that has yielded results for comparison to known thermodynamic quantities. Introducing the expression for the temperature dependence of the Gibbs free energy of fcc CoN provided by their study into the HSC program [20] we obtain for reaction (1) at room temperature the enthalpy (or the negative of the enthalpy of formation), entropy, Gibbs free energy, equilibrium constant and specific heat capacity. These are shown in table 1. Immediately it is clear that the negative output value for the reaction enthalpy corroborates the experimental report [10] that the dissociation reaction (1) is exothermic whilst the overall success of the calculations confirms that the reaction is feasible and directed to the right in equation (1) because of the negative Gibbs free energy 
Table 1. Thermodynamic parameters for the dissociation reaction (1) from [19] and using the HSC program [20]. $\Delta H_{\mathrm{r}}$ is the enthalpy of the reaction, $\Delta S$ is the entropy, $\Delta G_{\mathrm{r}}$ is the reaction Gibbs free energy, $K_{\mathrm{e}}$ is the equilibrium constant and $C_{p}$ is the specific heat capacity-all at $298.15 \mathrm{~K}$.

\begin{tabular}{lll}
\hline Parameter & Value & Units \\
\hline$\Delta H_{\mathrm{r}}$ & -22.375 & $\mathrm{~kJ} \mathrm{~mol}^{-1}$ \\
$\Delta S$ & -2.28 & $\mathrm{~J} \mathrm{~K}^{-1} \mathrm{~mol}^{-1}$ \\
$\Delta G_{\mathrm{r}}$ & -21.694 & $\mathrm{~kJ} \mathrm{~mol}^{-1}$ \\
$\lg \left(K_{\mathrm{e}}\right)$ & 3.801 & - \\
$C_{p}$ & 487 & $\mathrm{~J} \mathrm{~kg}^{-1} \mathrm{~K}^{-1}$ \\
\hline
\end{tabular}

and large equilibrium constant from which we also conclude that the reverse reaction is negligible. This is appropriate and expected for this simple model. Finally, but not shown in table 1 , the thermodynamic calculations of the reaction in (1) using the HSC program also showed that the enthalpy of the reaction $\Delta H_{\mathrm{r}}$ does not change significantly within the temperature range of interest in this work (300-1900 K).

\subsection{Reaction kinetics}

The thermodynamic calculations predict that reaction (1) should proceed even at room temperature. This is not, however, observed experimentally, indicating that the reaction is driven by the kinetics of the thermal processing applied and an approximate model to take account of this is now developed.

From the stoichiometry of the reaction in (1), the reaction rates $r=\mathrm{d} C / \mathrm{d} t$ in mol $\left(\mathrm{m}^{3} \mathrm{~s}\right)^{-1}$, where $C$ is the concentration in mole, of the different elements are given by:

$$
\frac{-r_{\mathrm{CoN}}}{1}=r_{\mathrm{Co}}=\frac{r_{\mathrm{N}_{2}}}{1 / 2} \text {. }
$$

Thus the rate of release of $\mathrm{N}_{2}, r_{\mathrm{N}_{2}}$ expressed in terms of the depletion rate of $\mathrm{CoN}$ is:

$$
r_{\mathrm{N}_{2}}=\frac{-1}{2} r_{\mathrm{CoN}}
$$

If the reaction is assumed essentially irreversible as indicated above, the rate depletion of $\mathrm{CoN}$ is proportional to its concentration $\mathrm{CoN}, C_{\mathrm{CoN}}$ :

$$
r_{\mathrm{CoN}}=-k_{\mathrm{r}} C_{\mathrm{CoN}}^{n}
$$

where $k_{\mathrm{r}}$ is the reaction rate constant $\left(\mathrm{s}^{-1}\right)$ and $n$ is the order of the reaction.

Ascribing an Arrhenian temperature dependence to the reaction, the rate constant may be expressed as:

$$
k_{\mathrm{r}}=A \exp \left(\frac{-E}{R_{\mathrm{g}} T}\right)
$$

in which $A$ is the frequency term $\left(1 \mathrm{~s}^{-1}\right), E\left(\mathrm{~J} \mathrm{~mol}^{-1}\right)$ the reaction activation energy, $R_{g}=8.3145\left(\mathrm{~J}(\mathrm{~mol} \mathrm{~K})^{-1}\right)$ the gas constant and $T(\mathrm{~K})$ the absolute temperature.

Now on substituting (3) and (4) into (2) we obtain the following expression for the rate of release of $\mathrm{N}_{2}$ during the reaction:

$$
r_{\mathrm{N}_{2}}=\frac{\mathrm{d} C_{\mathrm{N}_{2}}}{\mathrm{~d} t}=\frac{A}{2} \exp \left(\frac{-E}{R_{\mathrm{g}} T}\right) C_{\mathrm{CoN}}^{n}
$$

thereby allowing the rate of heat energy density release (or power density) due to the exothermic reaction, $Q_{\mathrm{r}}$, to be derived from:

$$
Q_{\mathrm{r}}=\Delta H_{\mathrm{r}} r_{\mathrm{N}_{2}}\left(\mathrm{~J}\left(\mathrm{~m}^{3} \mathrm{~s}\right)^{-1}\right)
$$

where $\Delta H_{\mathrm{r}}$ is the enthalpy of the reaction.

The rate equation (5) can be further simplified by first expressing the concentrations of the participating components in terms of the initial concentration $C_{\mathrm{CoN}}^{0}$ and converted molar fraction $x$ thus:

$$
\begin{gathered}
C_{\mathrm{CoN}}=C_{\mathrm{CoN}}^{0}-2 C_{\mathrm{N}_{2}}=(1-x) C_{\mathrm{CoN}}^{0} \\
C_{\mathrm{Co}}=x C_{\mathrm{CoN}}^{0} \quad C_{\mathrm{N}_{2}}=\frac{x}{2} C_{\mathrm{CoN}}^{0}
\end{gathered}
$$

when multiplying both sides of (5) by $2 / C_{\mathrm{CoN}}^{0}$ produces the rate equation as a function of a single variable $x$, the conversion fraction that is used in the simulations:

$$
\frac{\mathrm{d} x}{\mathrm{~d} t}=A(1-x)^{n} \exp \left(\frac{-E}{R_{\mathrm{g}} T}\right) .
$$

Unfortunately, to the best of our knowledge there are no published values for the activation energy or frequency factor pertinent to the dissociation of $\mathrm{CoN}$ so we must investigate other routes to obtain justifiable values for these parameters. Conventionally activation energies are obtained by measuring the temperature either at the peak of a reaction (identified for example from differential thermal or magneto-thermal analysis or by monitoring resistivity change) or of a transition in phase or state during isothermal heating experiments. These temperatures are a function of the heating rate and when measurements are available at several different rates the activation energy may be derived from the slope of the plot on applying a Kissinger [21] type analysis. However, in the case of the Co-N system this is not currently possible since the only reports $[13,14]$ of ramped heating experiments have been conducted at single heating rates which are, moreover, very low when compared to what is now intended. Instead we follow a simple approach that allows incorporation of the reaction kinetics into the proposed model by assuming the disassociation reaction is first order $(n=1)$ and occurs in a single step. Under these assumptions, the condition at the maximum reaction rate (at transition temperature $T_{\mathrm{r}}$ ) for a firstorder reaction can be written as [21]:

$$
\frac{\alpha E}{R T_{\mathrm{r}}^{2}}=A \exp \left(\frac{-E}{R T_{\mathrm{r}}}\right)
$$

and a solution to this nonlinear equation for the activation energy, $E$, is then possible by assuming known or appropriate values for $A$ and the annealing rate, $\alpha$ :

$$
E=R T_{\mathrm{r}} W\left(\frac{A T_{\mathrm{r}}}{\alpha}\right)
$$

where $W(\cdot)$ is the Lambert (or product $\log$ ) function which can be calculated numerically. Moreover, since $A$ and $\alpha$ are arguments of the Lambert function, large changes in $A$ or $\alpha$ have only modest effect on $E$, which is mainly proportional to 
Table 2. Calculated values of the activation energy for different transition temperatures using the condition of peak reaction from Kissinger analysis [21] and assuming a first-order reaction with a typical value for $A=10^{11} \mathrm{~s}^{-1}$ and a heating rate of $10 \mathrm{~K} \mathrm{~min}^{-1}$ from [14].

\begin{tabular}{lc}
\hline$T_{\mathrm{r}}(\mathrm{K})$ & $E\left(\mathrm{~kJ} \mathrm{~mol}^{-1}\right)$ \\
\hline 300 & 73.43 \\
350 & 86.11 \\
400 & 98.84 \\
450 & 111.62 \\
500 & 124.44 \\
550 & 137.31 \\
600 & 150.21 \\
650 & 163.15 \\
700 & 176.11 \\
\hline
\end{tabular}

$T_{\mathrm{r}}$. By taking $10^{11} \mathrm{~s}^{-1}$ as an appropriate value for $A$ (within the range $10^{10}-10^{14} \mathrm{~s}^{-1}$ from collision theory) and a heating rate $\alpha=0.167 \mathrm{~K} \mathrm{~s}^{-1}\left(=10 \mathrm{~K} \mathrm{~min}^{-1}\right)$ following [14] we are thus able to calculate $E$ for a range of $T_{\mathrm{r}}$ as shown in table 2 .

As previously discussed, the consensus from several studies is that the dissociation process is centred on a transition temperature of $573 \mathrm{~K}$ but can occur anywhere between 500 and $670 \mathrm{~K}$ depending on the specific thermal processing conditions. Therefore, in selecting kinetic parameters for use in subsequent modelling we choose an activation energy of $143 \mathrm{~kJ} \mathrm{~mol}^{-1}$ by averaging over this temperature range in table 2 .

\subsection{Electro-thermal model and Joule heating}

Localized processing of nanometre sized regions of $\mathrm{CoN}$ films may in principle be achieved by passing a current from the tip of a conductive AFM probe contacting the film's free surface to a highly conductive under-layer as shown in figure 1. This current will induce Joule heating in the small regions surrounding the tip contact area and drive the dissociation reaction described by (1). To study this localized heating process and its attendant reaction kinetics an electro-thermal model is developed to allow its realistic simulation. The model involves the simultaneous solution of a system of coupled equations comprising Laplace's equation to provide by way of the scalar potential a representative description of the electric field in the material stack shown in figure 2, the reaction rate equation (7) and the heat conduction equation to express the diffusion of energy produced by heating sources. Although the primary driving source is initially Joule heating, provision is made to include other source terms such as the identified exothermic nature of the reaction. Using temperature as an input, the model determines the molar fraction of the converted $\mathrm{CoN}$ material, $x$, and therefore concentrations of $\mathrm{CoN}, \mathrm{Co}$ and $\mathrm{N}_{2}$.

Laplace's equation for the scalar electric potential, $V$, in the geometry of figure 2 is:

$$
\nabla \cdot(\sigma \nabla V)=0
$$

where $\sigma$ is the electrical conductivity of the various materials in the structure. Solution of this equation in the $\mathrm{CoN}$ layer

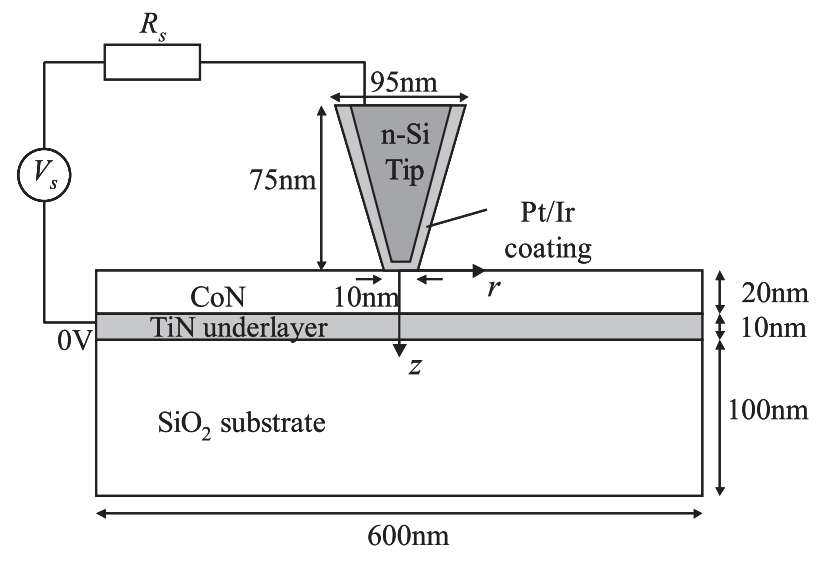

Figure 2. Probe heating system geometry and dimensions with the centre of the cylindrical coordinate system at the centre of the tip contact with the CoN layer. The Pt/Ir conductive tip coating is $5 \mathrm{~nm}$ thick. $V_{\mathrm{s}}$ is the applied voltage source and $R_{\mathrm{s}}$ is the series resistance in the system.

provides the power density source for Joule heating:

$$
Q_{\mathrm{J}}=\sigma|\nabla V|^{2}
$$

which is then used in the heat conduction equation:

$$
\nabla \cdot(k \nabla T)+\left(Q_{\mathrm{r}}+Q_{\mathrm{J}}\right)=\rho C_{p} \frac{\partial T}{\partial t}
$$

to determine the temperature distribution, $T$, in the material stack. In (10) $k$ is the thermal conductivity, $\rho$ is the density, $C_{p}$ is the specific heat and the heating sources include the Joule heating power density $Q_{\mathrm{J}}$ from (9), and the exothermic reaction enthalpy power density $Q_{\mathrm{r}}$ from (6).

\subsection{Numerical model}

Numerical modelling is implemented in the finite-element software Comsol Multiphysics. A three-dimensional geometry with cylindrical symmetry in which the centre of the coordinate system is located at the centre of the tip, as shown in figure 2 , forms the basis of the model. The medium stack is assumed to consist of a $20 \mathrm{~nm} \mathrm{CoN}$ film on a $10 \mathrm{~nm}$ TiN electrode backed by a $100 \mathrm{~nm} \mathrm{SiO}_{2}$ substrate to provide thermal and electrical insulation. The boundaries of the material stack in the radial direction are kept at room temperature $(300 \mathrm{~K})$ while the top and bottom boundaries are thermally insulated. Similarly, to simulate their effectively near infinite real extent, the thickness of the $\mathrm{SiO}_{2}$ substrate and the extent of the layers in the radial direction are made large compared to the thicknesses of the $\mathrm{CoN}$ and TiN layers and tip contact area so that their boundaries have negligible effect on the temperature distribution in the material stack.

A typical, commercially available, AFM probe fabricated from n-type silicon with a conductive and durable Pt/Ir coating is taken as the basis of the probe model. Its dimensions are as illustrated in figure 2 with a coating thickness of $5 \mathrm{~nm}$ and a $10 \mathrm{~nm}$ tip contact diameter. The radial boundaries of the tip are assumed thermally insulated, while the top of the probe is kept at room temperature to allow cooling of the heated region 
Table 3. Thermal and electrical parameters used in the numerical simulations. All parameters are at room temperature where appropriate.

\begin{tabular}{lclll}
\hline Material & $\begin{array}{l}\text { Density, } \rho \\
\left(\mathrm{kg} \mathrm{m}^{-3}\right)\end{array}$ & $\begin{array}{l}\text { Specific heat, } \\
C_{p}\left(\mathrm{~J} \mathrm{~kg}^{-1} \mathrm{~K}^{-1}\right)\end{array}$ & $\begin{array}{l}\text { Thermal conductivity, } \\
k\left(\mathrm{~W} \mathrm{~m}^{-1} \mathrm{~K}^{-1}\right)\end{array}$ & $\begin{array}{l}\text { Electrical conductivity, } \\
\sigma(\Omega \mathrm{m})^{-1}\end{array}$ \\
\hline Sb-doped Si tip & 2300 & 712 & 148 & 10000 \\
Pt90/Ir10 coating & 21560 & 133 & 31 & $4 \times 10^{6}$ \\
$\mathrm{CoN}$ & $4326^{\mathrm{a}}$ & $487^{\mathrm{b}}$ & $1.82^{\mathrm{c}}$ & $0.25 \times 10^{6 \mathrm{~d}}$ \\
$\mathrm{Co}$ & 8862 & 421 & 99.2 & $16 \times 10^{6}$ \\
$\mathrm{TiN}$ & 5400 & 545 & 20 & $5 \times 10^{6}$ \\
$\mathrm{SiO}_{2}$ & 2203 & 703 & 1.38 & $1 \times 10^{-14}$ \\
\hline
\end{tabular}

${ }^{\mathrm{a}}$ Reference [14]. ${ }^{\mathrm{b}}$ HSC program [20] using thermodynamic data from [19].

${ }^{\mathrm{c}}$ Calculated from the Wiedemann-Franz law at room temperature. ${ }^{\mathrm{d}}$ Reference [15].

in the CoN layer through the probe. Perfect thermal contact is assumed at the interfaces between different layers in the material stack for simplicity.

The probe, $\mathrm{CoN}$ and $\mathrm{TiN}$ layers are active regions in the electrical simulations but the $\mathrm{SiO}_{2}$, being an electrical insulator, does not disrupt the distribution of the electric fields produced by the tip and TiN layer. All boundaries of the electrically active regions in the figure are assumed electrical insulators with the exception of the top surface of the probe where a positive potential is applied with respect to the grounded TiN electrode to induce current flow in the $\mathrm{CoN}$ layer. Contact resistance between the tip and $\mathrm{CoN}$ layer is assumed negligible for simplicity.

Values taken for the electrical and thermal parameters of the different materials used in modelling the structures of figure 2 are listed in table 3 . Since the molar fraction of converted $\mathrm{CoN}$ changes as the reaction progresses the electrical and thermal conductivities of the CoN layer are functions of both position and time and the model includes provision for change in these parameters with progressive decomposition through:

$$
k=(1-x) k_{\mathrm{CoN}}+x k_{\mathrm{Co}} \quad \sigma=(1-x) \sigma_{\mathrm{CoN}}+x \sigma_{\mathrm{Co}} .
$$

The heat capacity and density are, however, included as functions of position only due to the nature of the Fourier heat conduction equation (10) used in the numerical solver. This is a reasonable assumption since the heat capacities of $\mathrm{CoN}$ and Co are not significantly different, as seen in table 3, and incorporation of the density change would require a more advanced model of a granular medium or a deformable mesh.

The initial conditions applied set the temperature of all the materials in the stack to room temperature and the initial conversion molar fraction of CoN, $x$, to zero.

Processing is initiated by the application of a voltage source, $V_{\mathrm{s}}$, applied between the tip and the TiN under-layer through a series resistor $R_{\mathrm{S}}$. This is included to limit the current and hence temperature rise in the $\mathrm{CoN}$ layer when its electrical conductivity increases during the decomposition process and nitrogen is released. This series resistor can also be used to extend the current model to include the physical resistance of the tip/cantilever and contact resistance values once these are available from experiment. The value of this series resistance here must be chosen to be less than the resistance of the $\mathrm{CoN}$ material before decomposition and greater than the resistance of the Co layer during the decomposition process. The resistances of the two phases may be estimated from the distribution of the tip electric field using the approximation from [22] for a circular conductor of radius $r_{\mathrm{c}}$ separated a distance $L$ from an infinitely conducting ground plate (discarding the effect of the fringing fields from the upper side of the circular conductor):

$$
R=\frac{1}{\sigma\left(\pi r_{\mathrm{c}}^{2} / L+2 r_{\mathrm{c}} \ln (4)\right)} .
$$

Thus for the CoN layer with $L=20 \mathrm{~nm}$ and a tip contact radius $r_{\mathrm{c}}=5 \mathrm{~nm}$, the resistance for the CoN phase with $\sigma=0.25 \times 10^{6}(\Omega \mathrm{m})^{-1}$ is calculated as $225 \Omega$, while the resistance of the Co phase with $\sigma=16 \times 10^{6}(\Omega \mathrm{m})^{-1}$ is $3.5 \Omega$. Hence a series resistor with $R_{\mathrm{S}}=10 \Omega$ was chosen to limit the current in the simulations.

The external circuit connections of the voltage source and series resistor to the electro-thermal probe model were implemented using the SPICE import feature in the AC/DC module of Comsol Multiphysics.

\section{Simulation results}

The electric field distribution and resulting temperature profile together with the reaction kinetics of the dissociation process directly determine the size and shape of the cobalt islands formed. These processes are complex functions not only of the geometry, dimensions, thermal and electrical properties of the various layers and materials in the stack but also of the voltage source profile [23]. Full parametric studies are consequently required to identify the optimum combination of material and source parameters that might ultimately produce the smallest cobalt islands commensurate with the requisite magnetic stability for ultra-high-density recording. However, the only variables used in the numerical modelling for this initial study are those describing the profile of the voltage source. The thicknesses of the CoN film are fixed on the basis of the experience gained from globally processing this material [5-7] and the other dimensions and materials in the structure shown in figure 2 are similarly pre-determined from practical considerations.

The profile of the voltage pulse $V_{\mathrm{s}}$ applied in the simulations is described by four parameters, its amplitude, duration and rise and fall times. These parameters were varied in a parametric study to identify values within an envelope limiting the maximum temperature in the $\mathrm{CoN}$ to values less 


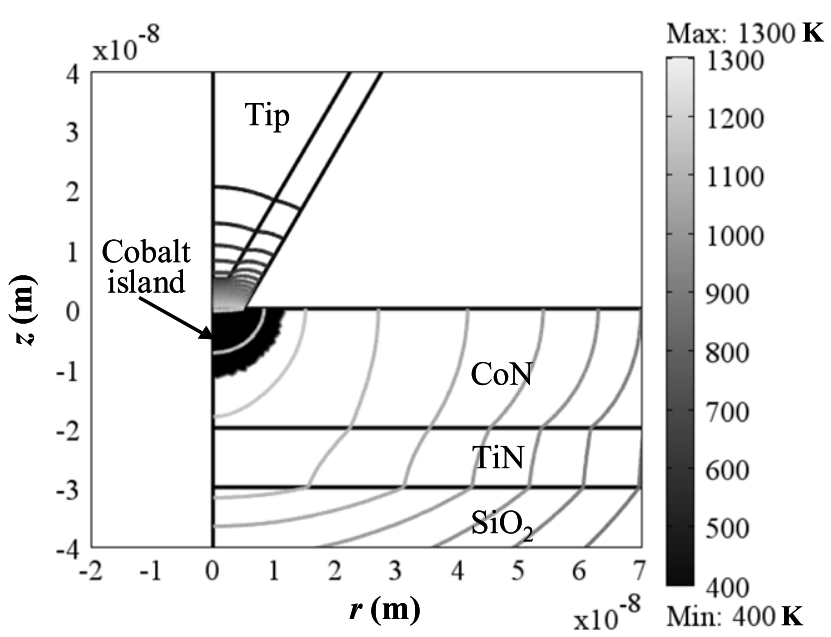

Figure 3. Output of probe processing simulations showing temperature contours and the formation of a $\mathrm{Co}$ island due to the local Joule heating of the CoN layer. An instant $8 \mu$ s from the start of the simulation is displayed with the Co region extent identified using $x>0.9$. The process continues to completion after $15 \mu \mathrm{s}$.

than the melting point of $(\sim 1900 \mathrm{~K}[19])$ whilst producing small islands of cobalt. Figure 3 shows the result of running the simulation when the electrical pulse applied through a $10 \Omega$ series resistance is described by input values of $1.2 \mathrm{~V}$ peak voltage, $10 \mu \mathrm{s}$ pulse width and $1 \mu \mathrm{s}$ for both rise and fall times of the voltage pulse. Identifying regions in which cobalt conglomerates following its freeing from the nitride with the spatial locations where $x>0.9$, the model predicts the production of cobalt islands with a form dictated by the symmetry of the high electric fields and the resulting hemispherical nature of the temperature contours in the region surrounding the tip contact area. An instant $8 \mu$ s from the start of the simulation is displayed. The process proceeds to completion in about $15 \mu \mathrm{s}$, predicting the production of hemispherical cobalt islands with radii $\sim 10 \mathrm{~nm}$ for the voltage source values used. Higher or lower applied voltages and/or much longer or shorter pulse widths would result in a corresponding increase or decrease in the radii of the islands formed.

The evolution of the applied voltage between the tip and TiN layer, the corresponding temperature in the $\mathrm{CoN}$ layer and the molar conversion fraction $x$ are plotted in figures 4(a) and (b) respectively. Figure 4(a) shows that the temperature initially increases with increasing voltage, reaching a peak value and starts to decrease. The decrease in temperature is attributed to the reduction in electric field and, and hence power density, in the heated region due to the increase in electrical conductivity caused by the dissociation reaction.

\section{Exploratory experimental measurements}

In light of the positive predictions of the model described above, tentative exploratory studies seeking experimental confirmation of the potential of localized probe processing have been conducted using a commercial (Veeco Innova) conductive mode AFM (C-AFM) and samples of cobalt nitride
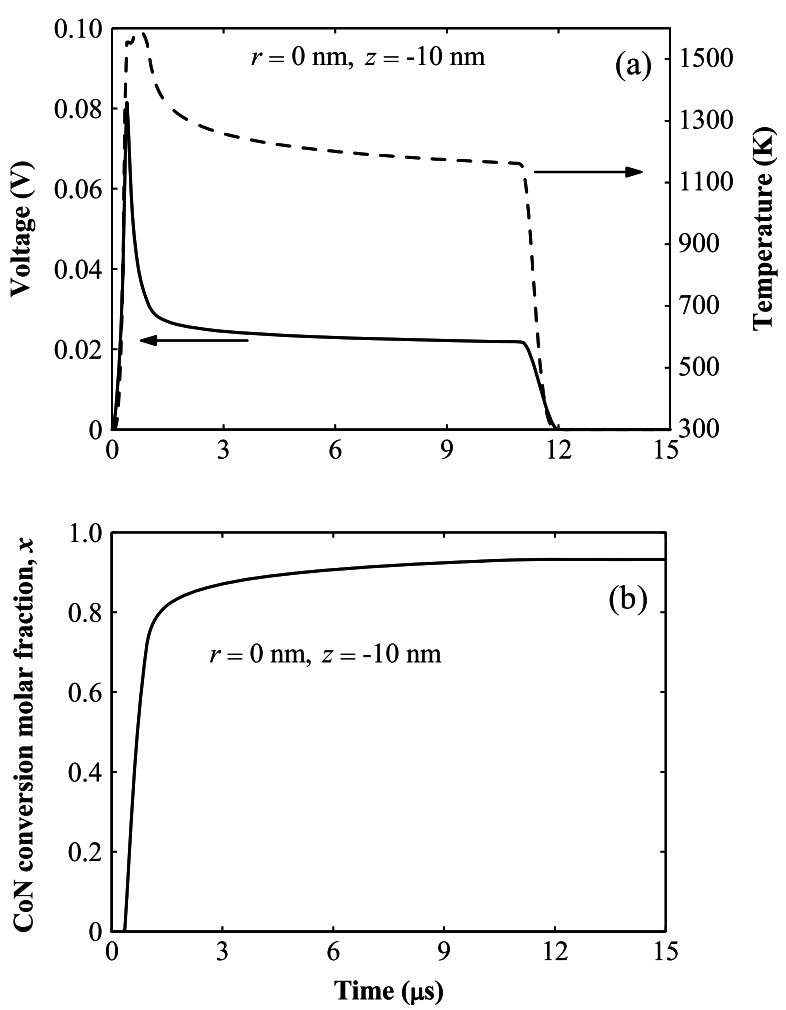

Figure 4. Time evolution of (a) voltage and temperature and (b) the molar fraction of converted $\mathrm{CoN}$ material with time at location $r=0 \mathrm{~nm}, z=-10 \mathrm{~nm}$ in the CoN layer. The voltage was taken between the tip and the TiN (ground) layer.

films prepared previously for global processing studies [5-8]. These are deposited on glass substrates and do not as yet possess the conductive TiN under-layer electrode shown in figure 2. Of necessity the return current path is therefore via a side surface electrode with the consequence that the exact form of the electric fields and resulting temperature contours are likely to be somewhat less confined or concentrated in the vicinity of the probe tip than portrayed in figure 3. Furthermore, the radius of the coated tips employed experimentally is in the range $30-50 \mathrm{~nm}$, considerably larger than assumed in the model. The thickness of the films, at $\sim 100 \mathrm{~nm}$, used for these initial experimental attempts at nano-scale processing, is also somewhat larger than in the simulation. The experimental results obtained reflect these differences; in general the features formed are somewhat larger than predicted by simulation. Figure 5 displays the result of scanning a tip continuously across a region of the film some $2 \mu \mathrm{m}$ square with a constant bias of $2 \mathrm{~V}$ applied to the surface electrode (tip at $0 \mathrm{~V}$ ) producing the increase in conductivity shown. It is not clear, however, that this level of bias is sufficient to fully release all nitrogen and progress the dissociation of $\mathrm{CoN}$ to a discontinuous layer of cobalt nanocrystallites, as observed previously during global processing. Topographically there is no evidence of the shrinkage in film structure observed in global processing and the small change in conductivity could simply be a consequence of and consistent with the partial processing of $\mathrm{CoN}$ to the intermediate phase $\mathrm{Co}_{2} \mathrm{~N}$, accompanied perhaps by a limited release of finely dispersed cobalt through the partially processed volume. 


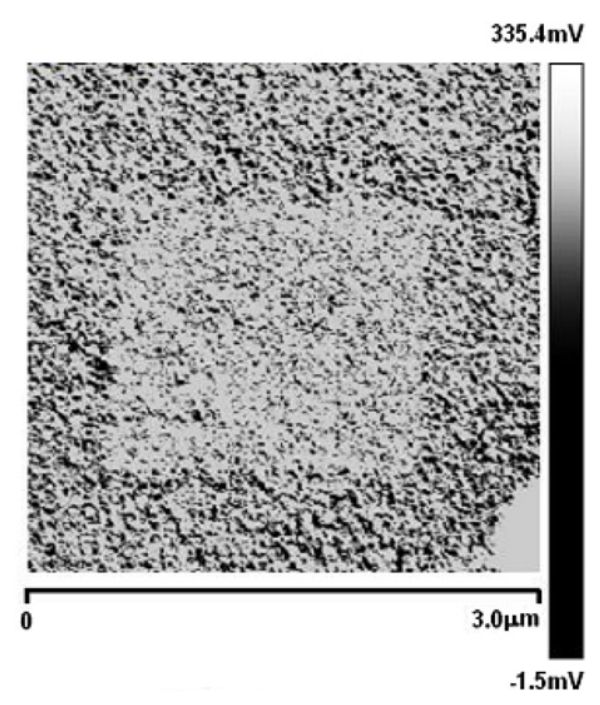

Figure 5. C-AFM scan showing the increase in conductivity produced on processing a $2 \mu \mathrm{m} \times 2 \mu \mathrm{m}$ square region (brighter region) of precursor film by scanning a tip with a bias of $2 \mathrm{~V}$ continuously across the surface. At this voltage there is no accompanying change in surface topology (see text).

The bias was therefore increased to $6 \mathrm{~V}$ and the conductivity changes this produced were accompanied by the topographical changes shown in figure 6(a). The upper portion of this figure shows the topography after the tip had been scanned continuously across the sample while applying a constant $6 \mathrm{~V}$ tip-sample bias to produce a rectangular area of processed material. The lower part of the figure shows the topography after the tip had been placed at five separate locations in turn and an $I-V$ curve (up to $10 \mathrm{~V}$ at $\sim 0.7 \mathrm{~V} \mathrm{~s}^{-1}$ ramp rate) taken at each one, so producing circular processed areas. The size of the first island obtained after $I-V$ testing is the largest (left-most island in row shown in bottom part of figure 6(a)), while all subsequent islands produced via the same $I-V$ testing routine are smaller and show a slight tendency to decrease in size (from left to right) as more and more $I-V$ curves were taken. This behaviour is most likely due to degradation of the tip's conductive coating caused by the relatively high voltages and currents present during the $I-V$ testing. It is well known that metal-coated AFM tips, such as the PtIr type used here, are not particularly robust and wear out (electrically) very rapidly, hence much recent interest in more robust $\mathrm{C}$-AFM tips, such as the PtSi and nano-diamond types [24, 25]. An $I-V$ curve recorded during the formation of one of the smaller islands of figure 6(a) is shown in figure 6(c). A consistent increase in conductivity at about $5 \mathrm{~V}$ followed by a subsequent near linear $I-V$ relationship was obtained in this and all other measured $I-V$ curves and is taken to indicate the production of metallic cobalt islands. However, as the medium is currently without a protective over-layer it is thought that, at the temperatures reached, the surface of the islands is immediately oxidized. This inference of surface oxidation is supported by the increase in surface topography of the processed regions in 6(a) and subsequent conductivity imaging as shown in figure 6(b). Evidence of an oxide layer $1.1 \mathrm{~nm}$ thick was also found previously [26] on $10 \mathrm{~nm}$ diameter
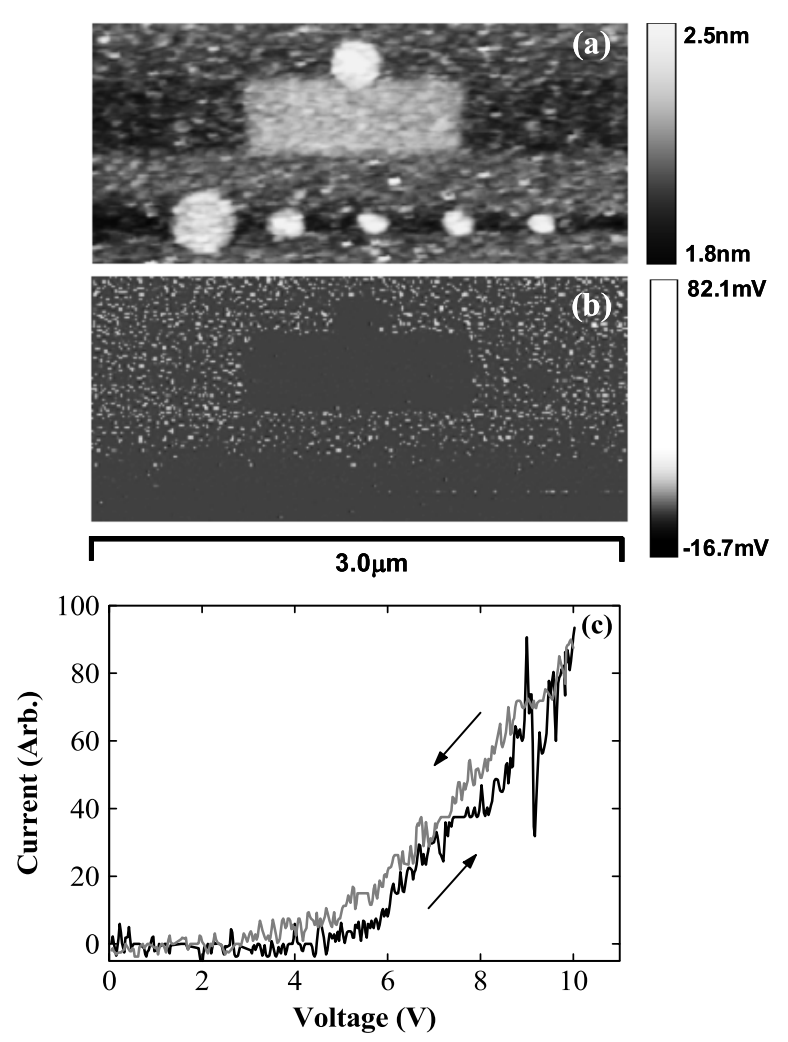

Figure 6. (a) AFM scan following electro-thermal processing of the sample. The upper portion of this figure shows the topography after the tip had been scanned continuously across the sample while applying a constant $6 \mathrm{~V}$ tip-sample bias to produce a rectangular area of processed material. The lower part of the figure shows the topography after the tip had been placed at five separate locations in turn and an $I-V$ curve (up to $10 \mathrm{~V}$ ) taken at each one, so producing circular processed areas. (b) C-AFM scan of the same region in (a) showing that the areas processed to cobalt rapidly acquire an insulating oxide surface (low conductivity, dark regions). (c) $I-V$ curve recorded during processing of precursor medium to produce one of the small islands in the sequence of processed regions in (a).

cobalt particles produced during studies of globally processed cobalt nitride films.

\section{Discussion}

The ideal outcome when nano-patterning a layer of precursor material, such as cobalt nitride, for application in ultrahigh areal density magnetic recording in a one-bit-per-particle format would be to realize the discrete magnetic elements in the form of cylindrical structures extending throughout its full thickness and arrayed normal to the surface. Attaining a geometrical structure of this nature would simultaneously maximize the packing density by presenting minimum crosssectional area at the recording surface and would ensure that each element has sufficient volume to produce the thermal stability required for data integrity and define, or contribute to defining, the anisotropy necessary to record in perpendicular mode with its many attendant advantages. However, it is clear from the simulations that in the simple cobalt nitride precursor system currently considered for the production of 
such ideal cylindrical structures is not immediately feasible. This is primarily a consequence of the relatively high electrical conductivity of the cobalt nitride which confines the tip electric fields to the surface of the material, enhancing resistive heating in the tip contact region to result in hemispherical forms that do not extend deep into the precursor layer. Of course and as already noted, higher voltages and longer pulse durations can increase the extent of the regions processed to cobalt further into the thickness of the cobalt nitride, but at the expense of increasing their diameter at the surface to detrimental effect on areal storage density. Similarly, while lower voltages and shorter pulse durations reduce the surface diameter of the processed regions they simultaneously reduce the volume of the material processed to cobalt with the consequent threat to magnetic stability at room temperature. In future, fuller parametric studies may make possible the design of a more complex media stack and electrode configuration that addresses the thermal budget constraints whilst providing an electric field path that better facilitates the production of columnar Co structures. At the same time provision can also be made to eliminate or minimize the impact of morphological changes resulting from the loss of nitrogen on surface topology. For the present we accept the limitations of the simple system examined and the predictions of the model in evaluating, in what follows, the potential of nano-probe processing for producing recording media.

As the simulation output of figure 3 makes clear, the application of profiled voltage pulses from a $10 \mathrm{~nm}$ diameter tip to the precursor media structure considered should easily produce isolated islands of cobalt with radii of around $10 \mathrm{~nm}$ or smaller. Assuming they retain values for saturation magnetization and anisotropy energy typical of cobalt (1422 $\mathrm{emu} \mathrm{cm}^{-3}$ and $4.5 \times 10^{6} \mathrm{ergs} \mathrm{cm}^{-3}$ respectively) the islands will remain thermally stable at the elevated temperatures common in recording systems, whilst exhibiting coercivities $(\approx 6 \mathrm{kOe}$ ) that enable recording using currently available heads. Arrayed on a regular grid with a common anisotropy direction, such locally patterned islands offer the potential for recording magnetic data at an areal density of around $1 \mathrm{Tbit} / \mathrm{in}^{2}$ in the one-bit-per-particle format. It has already been discussed how the island dimensions may be reduced by lowering the processing pulse profile and if more deterministic control over the anisotropy is then found necessary the application of a weak global magnetic field during processing may suffice. Even for the relatively low energy precursor medium based solely on cobalt presently considered, the reduction in island volume required to achieve a two-or three-fold increase in areal density would not significantly compromise the thermal stability of the medium. A $5 \mathrm{~nm}$ radius island would, for example, have a blocking temperature around $683 \mathrm{~K}$, a coercivity of approximately $3 \mathrm{kOe}$ and offer recording densities approaching $3 \mathrm{Tbit} / \mathrm{in}^{2}$. It therefore appears that for the present the dimensions of the probe tip rather than thermal stability remain the principal constraint on the achievable areal density.

Finally, to complete an evaluation of the potential of this processing format for recording purposes it is necessary to consider how its practical embodiment might be achieved.
We examine briefly two possible scenarios. The first is based on the 'Millipede' concept developed originally by IBM [27, 28]. This combines ultra-high areal density with useable data rates by employing an array of 1024 AFM type tips to access a rectangular data surface. Such a scheme might be copied to produce the equivalent of a magnetic hard-drive in a very small, near solid-state package in which formatting the drive would simultaneously produce the patterned medium. Ideally magnetic recording and readout in such a device might subsequently be by spin-torque transfer and magneto-resistive sensing, respectively, although this would require the incorporation of additional layers both magnetic and non-magnetic and include replacing the PtIr probe tip coating with an appropriate magnetic medium. There is considerable evidence in the literature supporting the feasibility of recording in this manner. Krause et al [29] have, for example, demonstrated in-plane magnetization switching of single domain $\mathrm{Fe}$ islands near the super-paramagnetic limit by applying current through the tip of a spin-polarized scanning tunnelling microscope (SP-STM). Similarly Wang et al [30] have simultaneously reversed the magnetization in clusters of cobalt particles less than $5 \mathrm{~nm}$ in diameter using a probe with a contact area approximately $30 \mathrm{~nm}$ in diameter. The case of spin-torque induced reversal of magnetization normal to the film plane has also been studied by Mangin et al [31] in nano-pillars composed of multilayer elements of $\mathrm{Ni} / \mathrm{Co}$ and/or Pt/Co. Applied on this same basis to a 3 Tbit/in ${ }^{2}$ recording medium comprised of cobalt islands formed by the probe processing route described in this paper indicates a critical switching current requirement of the order of a few $\mathrm{mA}$, which is entirely practicable. It should also be pointed out that a particular advantage of carrying out the processing (patterning) of media by the same probe array that performs the writing and reading is that the patterned 'bits' and the write/read tips are automatically aligned with each other. Such alignment would be hard to achieve by conventional lithographic techniques assuming (as is usually the case) that the tip array and the media patterning were via separate lithographic processes; in this case each tip in the probe array would require its own $x-y$ actuation system to ensure that all tips in the array were simultaneously colocated over patterned regions, significantly increasing the cost and complexity of array fabrication. A potential disadvantage of the probe processing route is, however, the relatively long processing time per particle. For example, assuming a capacity of 4 Tbit, the minimum that might currently be considered worthwhile developing, would require approximately $9 \mathrm{~h}$ of processing with a 1024 tip array using the pulse times of section $3(8 \mu \mathrm{s})$. No doubt such a long processing time could be reduced significantly by proper optimization of the medium stack, processing voltage etc. It might also be feasible in a practical system to spread the processing time over a number of device accesses (i.e. selectively format regions only when necessary to release their capacity), so that an excessively long initial format time is not required. Notwithstanding such improvements, it is nevertheless prudent to consider alternative electro-thermal patterning routes. One exciting possibility is to use a form of electro-thermal nano-imprinting to pattern a 
'conventional' hard-disk platter coated with a cobalt nitride (or other suitable) precursor layer. In this approach an electrically conducting patterned template would be brought into contact with the precursor material and a voltage applied between an electrode layer underneath the precursor and the template itself. The patterned regions on the template thus perform electro-thermal processing in a similar manner to the probe tip described above, replicating the entire template pattern on the disk platter in a single step.

Finally, it should be noted that although discussions in this paper have concentrated on $\mathrm{CoN}$ based media (since this is the simplest system and the one that has been most extensively studied to date), the electro-thermal processing methods described are potentially extendable to the production of a wide variety of magnetic materials (e.g. PtCo, FeCo, NiFe alloys) for a wide variety of applications.

\section{Acknowledgment}

The authors wish to thank Dr Stan Ashcroft for helpful discussions on the thermodynamic analysis in this work.

\section{References}

[1] Barmak K, Kim J and Shell S 2002 Appl. Phys. Lett. $804268-70$

[2] Wood R, Williams M, Kavcic A and Miles J 2009 IEEE Trans. Magn. 10 917-23

[3] Kryder M H, Gage E C, McDaniel T W, Challener W A, Rottmayer R E, Ju G, Hsia Y and Erden F 2008 IEEE Proc. $961810-35$

[4] Zhu J G, Zhu X and Tang Y 2008 IEEE Trans. Magn. 44 125-31

[5] Carey R, Newman D M and Holmes B 1999 J. Appl. Phys. 85 5537-9

[6] Carey R, Newman D M and Holmes B 1999 J. Magn. Soc. Japan 2335

[7] Carey R, Newman D M and Holmes B 2000 IEEE Trans. Magn. 36 3012-4

[8] Jollie M, Choo D, Newman D M, Wears M L and Miles J 2003 IEE Proc. Sci. Meas. Technol. 150 240-3
[9] Newman D M, Wears M L and Jollie M 2004 International Patent Application Number: PCT/GB2004/002118 Nano-particulate medium for UHD perpendicular recording

[10] Newman D M, Wears M L, Jollie M and Choo D 2007 Nanotechnology $\mathbf{1 8} 205301$

[11] Matsuoka M, Ono K and Inukai T 1987 IEEE Trans. Magn. 23 2788-90

[12] Oda K, Yoshio T and Oda K 1987 J. Mater. Sci. 22 2729-33

[13] Suzuki K, Kaneko T, Yoshida H, Morita H and Jujimori H 1995 J. Alloys Compounds 224 232-6

[14] Maya L, Paranthaman M, Thomson J, Thundat T and Stevenson R 1996 J. Appl. Phys. 79 7905-10

[15] Fang J, Yang L, Hsu C, Chen G, Lin Y and Chen G 2004 J. Vac. Sci. Technol. A 22 698-704

[16] Atkins P and De Paula J 2006 Physical Chemistry 8th edn (New York: Oxford University Press) p 54

[17] For $\mathrm{Co}_{2} \mathrm{~N}, \mathrm{Co}_{4} \mathrm{~N}$ and $\mathrm{CoN}$ Group IV Physical Chemistry: Ca-Cd-Co-Zr 1993 (Landolt-Börnstein vol 5c) (New York: Springer) pp 1-3

[18] For $\mathrm{Co}_{3} \mathrm{~N}$ Wagman D D, Evans W H, Parker V P, Halow I, Bailey S M and Schumn R H 1969 Selected Values of Chemical Thermodynamic Properties (Washington, DC: National Bureau of Standards Technical Note) pp 270-4 Barin I, Knacke O and Kubaschewski O 1973 Thermodynamic Properties of Inorganic Substances (New York: Springer) Supplement 1977

[19] Guillermet A F and Jonsson S 1992 Z. Metallk. 83 21-31

[20] Roine A 2002 Outokumpu HSC Chemistry ${ }^{\circledR}$ for Windows Outokumpu Research Oy, Finland www.outokumpu.com/hsc

[21] Kissinger H 1957 Anal. Chem. 29 1702-6

[22] Sequin C H 1971 Solid-State Electron. 14 417-20

[23] Wright C D, Armand M and Aziz M M 2006 IEEE Trans. Nanotechnol. 5 50-61

[24] Bhaskaran H, Sebastian A, Dreschsler U and Despont M 2009 Nanotechnology 20105701

[25] Oliver R A 2008 Rep. Prog. Phys. 71076501

[26] Holmes B M, Newman D M and Wears M L 2007 J. Magn. Magn. Mater. 315 39-45

[27] Vettiger P et al 2002 IEEE Trans. Nanotechnol. 139-55

[28] Pantazi A et al 2008 IBM J. Res. Dev. 52 493-511

[29] Krause S, Berbil-Bautista L, Herzog G, Bode M and Wiesndanger R 2007 Science 317 1537-40

[30] Wang X J, Zou H and Ji Y 2008 Appl. Phys. Lett. 93162501

[31] Mangin S, Ravelosona D, Katine J A, Cary M J, Terris B D and Fullerton E 2006 Nat. Mater. 5 210-5 